\title{
Short note on a new arithmetic function
}

\author{
Krassimir T. Atanassov \\ Department of Bioinformatics and Mathematical Modelling \\ Institute of Biophysics and Biomedical Engineering \\ Bulgarian Academy of Sciences \\ Acad. G. Bonchev Str., B1. 105, Sofia-1113, Bulgaria \\ e-mail: kratebas.bg
}

Received: 4 July 2016

Accepted: 29 October 2017

Abstract: A new arithmetic function is introduced. It is illustrated with some examples with well known arithmetic functions, as, e.g., $\pi$ - and $\varphi$-functions.

Keywords: Arithmetic functions $\varphi, \psi$ and $\sigma$.

2010 Mathematics Subject Classification: 11A25.

First, let for every arithmetic function $F$ such that for every natural number $n \geq 1, F(n) \leq n$, we define:

1. $F_{0}(n)=n$,

2. $F_{1}(n)=F(n)$,

3. for every $k \geq 1: F_{k+1}(n)=F\left(F_{k}(n)\right)$.

Second, let us define the following new arithmetic function $f_{F}$, where $F$ is the function, defined above, such that for every natural number $n \geq 2$ :

$$
f_{F}(n)=k \text { if and only if for } k \geq 1, F_{k-1}(n)>1 \text { and } F_{k}(n)=1
$$

For example, if $F$ is the function $\pi$, determining the number of primes that are less than or equal to $n$ (see, e.g. $[3,4]$ ), then the values of the new function are given in the following table. 
Table 1

\begin{tabular}{|r|r|r||r|r|r||r|r|r||r|r|r|}
\hline$n$ & $\pi(n)$ & $f_{\pi}(n)$ & $n$ & $\pi(n)$ & $f_{\pi}(n)$ & $n$ & $\pi(n)$ & $f_{\pi}(n)$ & $n$ & $\pi(n)$ & $f_{\pi}(n)$ \\
\hline 1 & 1 & 1 & 9 & 4 & 3 & 126 & 30 & 5 & 5380 & 708 & 7 \\
2 & 1 & 1 & 10 & 4 & 3 & 127 & 31 & 6 & 5381 & 709 & 8 \\
3 & 2 & 2 & 11 & 5 & 4 & 128 & 31 & 6 & 5382 & 709 & 8 \\
4 & 2 & 2 & $\vdots$ & $\vdots$ & $\vdots$ & $\vdots$ & $\vdots$ & $\vdots$ & $\vdots$ & $\vdots$ & $\vdots$ \\
5 & 3 & 3 & 30 & 10 & 4 & 708 & 126 & 6 & 52710 & 5380 & 8 \\
6 & 3 & 3 & 31 & 11 & 5 & 709 & 127 & 7 & 52711 & 5381 & 9 \\
7 & 4 & 3 & 32 & 11 & 5 & 710 & 127 & 7 & 52712 & 5381 & 9 \\
8 & 4 & 3 & $\vdots$ & $\vdots$ & $\vdots$ & $\vdots$ & $\vdots$ & $\vdots$ & $\vdots$ & $\vdots$ & $\vdots$ \\
\hline
\end{tabular}

For the natural number

$$
n=\prod_{i=1}^{k} p_{i}^{\alpha_{i}}
$$

where $k, \alpha_{1}, \ldots, \alpha_{k}, k \geq 1$ are natural numbers and $p_{1}, \ldots, p_{k}$ are different primes, the following arithmetic functions are defined by:

$$
\varphi(n)=\prod_{i=1}^{k} p_{i}^{\alpha_{i}-1}\left(p_{i}-1\right), \varphi(1)=1
$$

(see, e.g. $[3,4])$ and

$$
\rho(n)=\prod_{i=1}^{k}\left(p_{i}^{\alpha_{i}}-p_{i}^{\alpha_{i}-1}+\ldots+p_{i}^{0}(-1)^{\alpha_{i}}\right), \rho(1)=1
$$

$($ see $[1,2])$.

Let

$$
\Phi(n)=\max _{1 \leq k \leq n} \varphi(n) .
$$

Theorem 1. For every natural number $n \geq 2$

$$
f_{\pi}(n) \leq f_{\Phi}(n) .
$$

Proof. For $n=2$ the assertion is valid, because $f_{\pi}(2)=1=f_{\Phi}(2)$. Let us assume that Theorem 1 is valid for some natural number $n$. We will prove it for $n+1$.

For number $n+1$ there are two cases.

If $n+1$ is a prime number, then

$$
f_{\pi}(n+1)=f_{\pi}(n)+1
$$

(by induction assumption)

$$
\leq f_{\Phi}(n)+1=f_{\Phi}(n+1) .
$$


If $n+1$ is not a prime number, then

$$
f_{\pi}(n+1)=f_{\pi}(n)
$$

(by (2) and induction assumption)

$$
\leq f_{\Phi}(n)=f_{\Phi}(n+1) \text {. }
$$

Let

$$
P(n)=\max _{1 \leq k \leq n} \rho(n) .
$$

Theorem 2. For every natural number $n \geq 2$

$$
f_{P}(n)=f_{\Phi}(n) .
$$

The proof of (3) is similar than the above one.

Function $F$ can have more than one argument. For example, let for the two natural numbers $n \geq 1, s \geq 2$ :

1. $F_{0}(n, s)=n$,

2. $F_{1}(n, s)=\left[\frac{n}{s}\right]$,

3. for every $k \geq 1: F_{k+1}(n, s)=F\left(F_{k}(n, s)\right)$.

Then for $f_{F}$ the following theorem is valid.

Theorem 3. For every natural numbers $n \geq 1, s \geq 2$ and for the above defined function $F$ :

$$
f_{F}(n, s) \leq \log _{s} n
$$

Obviously, the equality exists for the case $n=s^{k}$ for some natural number $k$.

\section{References}

[1] Atanassov, K. (2001) Remarks on $\varphi, \sigma, \psi$ and $\rho$ functions. Notes on Number Theory and Discrete Mathematics, 7, 1, 1-3.

[2] Atanassov, K. (2009) A remark on an arithmetic function. Part 2. Notes on Number Theory and Discrete Mathematics, 15, 3, 21-22.

[3] Mitrinović, D., \& Sándor, J. (1996) Handbook of Number Theory, Kluwer Academic Publishers.

[4] Nagell, T. (1950) Introduction to Number Theory, John Wiley \& Sons, New York. 